\title{
REMARKS ON PARTITIONER ALGEBRAS
}

\author{
ALAN DOW AND RYSZARD FRANKIEWICZ
}

(Communicated by Andreas R. Blass)

\begin{abstract}
Partitioner algebras are defined in [1] and are a natural tool for studying the properties of maximal almost disjoint families of subsets of $\omega$. We answer negatively two questions which were raised in [1]. We prove that there is a model in which the class of partitioner algebras is not closed under quotients and that it is consistent that there is a Boolean algebra of cardinality $\aleph_{1}$ which is not a partitioner algebra.
\end{abstract}

For any almost disjoint family $\mathfrak{M}$ of subsets of $\omega$, a set $X \subset \omega$ is a partitioner of $\mathfrak{M}$ if $(\forall M \in \mathfrak{M}) \quad\left(M \subset^{*} X \vee M \cap X={ }^{*} \varnothing\right)$. Recall that for sets $A$ and $B, A \subset^{*} B$ if the set $A \backslash B$ is finite and $A={ }^{*} B$ if $A \subset^{*} B$ and $B \subset^{*} A$. The set of partitioners of $\mathfrak{M}$ forms a Boolean algebra $\mathfrak{P}_{\mathfrak{M}}$. Let $\mathfrak{I}_{\mathfrak{M}}$ be the ideal in $\mathfrak{B}_{\mathfrak{M}}$ generated by $\mathfrak{M}$. The partitioner algebra $\mathfrak{B}_{\mathfrak{M}}$, corresponding to $\mathfrak{M}$ is then the quotient $\mathfrak{P}_{\mathfrak{M}} / \mathfrak{I}_{\mathfrak{M}}$. An algebra $\mathfrak{B}$ is said to be representable if it is isomorphic to a partitioner algebra for some mad (maximal almost disjoint) family $\mathfrak{M}$. Similarly an algebra $\mathfrak{B}$ is said to be weakly representable if it is embeddable in a representable algebra. We will say that a set $Y$ splits a set $M$ if $M \cap Y$ and $M \backslash Y$ are both infinite. Note that $Y \notin \mathfrak{B}_{\mathfrak{M}}$ if and only if $Y$ splits some member of $\mathfrak{M}$. See [1] for more details. If $S$ is any countably infinite set, we generalize each of the above notions to apply to subsets of $S$ in the obvious manner.

\section{HOMOMORPHIC IMAGES}

In this section we show that it is consistent with Martin's Axiom that the class of representable algebras is not closed under homomorphic images. It is shown in [1] that it follows from $\mathrm{CH}$ that every algebra of cardinality $\mathfrak{c}$ is representable.

Recall that $\mathfrak{b}$ is the minimum cardinality of an unbounded (mod finite) family of functions from $\omega$ to $\omega$. We let $\mathscr{P}(X)$ denote the power set of $X$. For a set $X$, a family $\mathfrak{S} \subset \mathscr{P}(X)$ is splitting if it splits every infinite $Y \subset X$. Then $\mathfrak{s}$ is the minimum cardinality of a family of subsets of $\omega$ which

Received by the editors May 29, 1990 and, in revised form, July 20, 1990.

1980 Mathematics Subject Classification (1985 Revision). Primary 03E35; Secondary 54B15, $54 \mathrm{D} 80$.

Research of the first author supported by NSERC of Canada. 
is splitting. A family $\mathfrak{A} \subset \mathscr{P}(X)$ is independent if for each disjoint pair of finite subfamilies $\mathfrak{H}$ and $\mathfrak{J}$ of $\mathfrak{A}, \cap \mathfrak{H} \cap \bigcap_{J \in \mathfrak{J}}[X-J]$ is infinite.

Theorem 1. If $\mathfrak{b} \leq \mathfrak{s}$, then the free algebra with $\mathfrak{b}$ generators is weakly representable.

Proof. Fix an unbounded family $\left\{f_{\alpha} \mid \alpha<\mathfrak{b}\right\} \subset{ }^{\omega} \omega$ of increasing functions and an independent family $\left\{A_{\alpha} \mid \alpha<\mathfrak{b}\right\} \subset \mathscr{P}(\omega)$. We assume also that for $\alpha<\beta<\mathfrak{b},\left\{n \mid f_{\alpha}(n) \geq f_{\beta}(n)\right\}$ is finite. For each $\alpha<\mathfrak{b}$, define a subset of $\omega \times \omega$,

$$
X_{\alpha}=\left[A_{\alpha} \times \omega\right] \backslash\left[\left\{(n, m) \mid m<f_{\alpha}(n)\right\}\right] .
$$

Claim. There is a maximal almost disjoint family $\mathfrak{M}$ of infinite subsets of $\omega \times \omega$ such that $\left\{X_{\alpha} \mid \alpha<\mathfrak{b}\right\} \subset \mathfrak{P}_{\mathfrak{M}}$.

Proof of Claim. Let $Y$ be an infinite subset of $\omega \times \omega$; we will show that there is an infinite subset of $Y$ which is not split by the family $\left\{X_{\alpha} \mid \alpha<\mathfrak{b}\right\}$. Indeed, if there is an $n$ such that $Y \cap(\{n\} \times \omega)$ is infinite, then this is trivial. On the other hand, if $Y$ is finite in each "column," we define $g \in{ }^{\omega} \omega$ such that for each $n \in$ $\omega, Y \cap[\{n\} \times \omega] \subset\{n\} \times g(n)$. Now choose $\beta<\mathfrak{b}$, such that $\left\{n \mid g(n)<f_{\beta}(n)\right\}$ is infinite (since $\left\{f_{\beta} \mid \beta<\mathfrak{b}\right\}$ is unbounded). Let $Y^{\prime}=\left\{(n, m) \in Y \mid m<f_{\beta}(n)\right\}$. Since we are assuming $\mathfrak{b} \leq \mathfrak{s}$, the family $\left\{X_{\alpha} \cap Y^{\prime} \mid \alpha<\beta\right\}$ is not a splitting family on $\mathscr{P}\left(Y^{\prime}\right)$. Therefore there is an infinite $M \subset Y^{\prime} \subset Y$ which is not split by this family. This completes the proof of the claim, since $X_{\gamma} \cap Y^{\prime}$ is finite for $\gamma>\beta$.

Therefore we may choose a maximal almost disjoint family $\mathfrak{M}$ of subsets of $\omega \times \omega$ so that no member of $\mathfrak{M}$ is split by any $X_{\alpha}$. Therefore $\left\{X_{\alpha} \mid \alpha<\mathfrak{b}\right\} \subset$ $\mathfrak{P}_{\mathfrak{M}}$. Since $\left\{X_{\alpha} \mid \alpha<\mathfrak{b}\right\}$ is an independent family, it follows that no infinite member of the algebra generated by it is in $\mathfrak{I}_{\mathfrak{M}}$ (although this would be easy to guarantee by refining $\mathfrak{M}$ ). Clearly, then $\mathfrak{B}_{\mathfrak{M}}$ contains the free algebra on $\mathfrak{b}$ generators.

We will need the following result.

Proposition 2 [3]. It is consistent with $M A+\mathfrak{c}>\aleph_{1}$, that $\mathscr{P}\left(\omega_{1}\right)$ is not representable.

We can now solve Problem 2 from [1].

Corollary 3. It is consistent with $M A$, that there is a representable algebra with a homomorphic image which is not representable.

Proof. Since $M A+\mathfrak{c}>\aleph_{1}$ implies that $2^{\aleph_{1}}=\mathfrak{c}$, it also implies that $\mathscr{P}\left(\omega_{1}\right)$ is a homomorphic image of the free algebra on $\mathfrak{c}$ generators. Let $\mathfrak{M}$ be chosen as in Theorem 1 so that $\mathfrak{B}=\mathfrak{B}_{\mathfrak{M}}$ contains the free algebra on $\mathfrak{c}$ generators as a subalgebra. Call this subalgebra $\mathfrak{C}$ and let $\varphi$ be the homomorphism to $\mathscr{P}\left(\omega_{1}\right)$. For each $\xi<\omega_{1}$, fix an ultrafilter $\mathfrak{U}_{\xi}$ on $\mathfrak{B}$ so that $B \in\left(\mathfrak{U}_{\xi} \cap \mathfrak{C}\right) \Rightarrow \xi \in \varphi(B)$. Now define a function $\psi$ from $\mathfrak{B}$ to $\mathscr{P}\left(\omega_{1}\right)$ by $\psi(B)=\left\{\xi \in \omega_{1} \mid B \in \mathfrak{U}_{\xi}\right\}$. 
We claim that $\psi$ is a homomorphism from $\mathfrak{B}$ onto $\mathscr{P}\left(\omega_{1}\right)$. To see that it is onto, let $Y \in \mathscr{P}\left(\omega_{1}\right)$ and note that each $B \in \varphi^{-1}(Y)$ maps onto $Y$ by $\psi$.

This completes the proof.

\section{NONREPRESENTABLE ALGEBRAS OF SIZE $\aleph_{1}$}

In this section we prove that if the splitting number, $\mathfrak{s}$, is less than $\mathfrak{b}$ then there is an algebra of size $\mathfrak{s}$ which cannot be represented. It has been shown to be consistent that $\mathfrak{s}=\aleph_{1}<\mathfrak{b}=\aleph_{2}$ in [4]. We will also show that under these hypotheses, $\mathscr{P}(\omega)$ cannot be represented.

Theorem 4. If $\mathfrak{s}<\mathfrak{b}$, the algebra $\mathfrak{B}$ (generated by a small splitting family together with the finite sets) is not even weakly representable.

Proof. Assume that $\mathfrak{s}<\mathfrak{b}$. Let $\left\{A_{\alpha} \mid \alpha \in \mathfrak{s}\right\} \subset \mathscr{P}(\omega)$ be a splitting family. Let $\mathfrak{B}$ be the subalgebra of $\mathscr{P}(\omega)$ generated by the splitting family together with the finite sets. We shall show that $\mathfrak{B}$ cannot be represented.

Indeed, suppose that $\mathfrak{M}$ is a mad family which represents $\mathfrak{B}$. Let $\varphi$ denote the embedding of $\mathfrak{B}$ into $\mathfrak{B}_{\mathfrak{M}}$.

Let $X_{0}=\varphi(\{0\})$ and for $n \in \omega$, let

$$
X_{n+1}=\varphi(\{n+1\}) \backslash\left[\bigcup_{k \leq n} X_{k}\right] \text {. }
$$

Since $\varphi$ is an embedding, for each $b \in \mathfrak{B}$, there is a sequence $\{I(b, n) \mid n \in$ $\omega\} \subset \mathfrak{I}_{\mathfrak{M}}$, such that

$$
n \in b \Rightarrow X_{n} \backslash \varphi(b) \subset I(b, n)
$$

and

$$
n \notin b \Rightarrow X_{n} \cap \varphi(b) \subset I(b, n) .
$$

Since the minimum cardinality of a maximal almost disjoint family of subsets of $\omega, \mathfrak{a}$, is at least $\mathfrak{b}$, we may choose $J_{n} \subset X_{n}$, such that $J_{n} \in \mathfrak{M}$ and $J_{n} \cap I(b, n)$ is finite for each $b \in \mathfrak{B}$.

Hence, for each $\alpha<\mathfrak{s}$, there is an $f_{\alpha} \in{ }^{\omega} \omega$, so that

$$
n \in A_{\alpha} \Rightarrow \varphi\left(A_{\alpha}\right) \supset J_{n} \backslash f_{\alpha}(n),
$$

and

$$
n \notin A_{\alpha} \Rightarrow \varphi\left(A_{\alpha}\right) \cap\left(J_{n} \backslash f_{\alpha}(n)\right)=\varnothing .
$$

Since $\mathfrak{s}<\mathfrak{b}$, the family $\left\{f_{\alpha}: \alpha<\mathfrak{s}\right\}$ is bounded in ${ }^{\omega} \omega$, so we may choose $h \in{ }^{\omega} \omega$, so that $f_{\alpha}<{ }^{*} h$ for each $\alpha<\mathfrak{s}$. Now we may choose $M \in$ $\mathfrak{M} \backslash\left\{J_{n}: n \in \omega\right\}$ so that $M \cap \bigcup_{n \in \omega} J_{n}-h(n)$ is infinite. Let $A=\left\{n \mid J_{n} \cap M \neq \varnothing\right\}$ and choose $\alpha<\mathfrak{s}$ so that $A_{\alpha}$ splits $A$-that is, $\left|A \cap A_{\alpha}\right|=\left|A \backslash A_{\alpha}\right|$. We claim that $\varphi\left(A_{\alpha}\right)$ splits $M$, which contradicts the fact that $\varphi\left(A_{\alpha}\right)$ is a partitioner. Indeed, let $m \in \omega$ be such that $h(n)>f_{\alpha}(n)$ for each $n \geq m$. Now it is routine to verify that

$$
\varphi\left(A_{\alpha}\right) \cap M \supset \bigcup_{n \in(A-m) \cap A_{\alpha}}\left[M \cap J_{n}\right],
$$


and

$$
M \backslash \varphi\left(A_{\alpha}\right) \supset \bigcup_{n \in(A-m) \backslash A_{\alpha}}\left[M \cap J_{n}\right]
$$

which are both infinite.

We now answer Problem 4 from [1].

Corollary 5. $\mathfrak{s}=\aleph_{1}<\mathfrak{b}$ implies there is an algebra of cardinality $\aleph_{1}$ which is not representable.

It turns out that this provides another model in which the solution to Problem 2 of [1] is negative. It is proven in [2] that the free algebra with $\aleph_{1}$ generators is representable. Therefore we obtain the following result as a corollary.

Corollary 6. If $\mathfrak{s}=\aleph_{1}<\mathfrak{b}$, then the class of representable algebras is not closed under homomorphic images.

Let us also remark that the above proof also proves the following.

Corollary 7. If $\mathfrak{s}<\mathfrak{b}$, then $\mathscr{P}(\omega)$ is not weakly representable.

\section{QUESTIONS}

(1) If $\kappa<\mathfrak{s}$, then the free algebra with $\kappa$ generators is easily seen to be weakly representable-is it representable?

(2) Is the free algebra on $\mathfrak{s}$ generators always weakly representable?

(3) Does the representability of $\mathscr{P}(\omega)$ imply that $\mathfrak{s}=\mathfrak{c}$ ? (It does not imply that $\mathfrak{p}=\mathfrak{c}$.)

(4) Does the representability of $\mathscr{P}(\omega)$ imply that $\mathscr{P}(\omega) /$ fin is representable?

(5) Does the representability of $\mathscr{P}(\omega)$ imply that all algebras of size at most $c$ are representable?

(6) Can Theorem 4 be proven even if the algebra $\mathfrak{B}$ is not made to be atomic?

\section{REFERENCES}

1. James E. Baumgartner and Martin Weese, Partition algebras for almost-disjoint families, Trans. Amer. Math. Soc. 274 (1982), 619-630.

2. A. Dow and P. Nyikos, Compact Hausdorff spaces with moderately large families of convergent sequences, in preparation.

3. R. Frankiewicz and P. Zbierski, On partitioner-representability of Boolean algebras, Fund. Math. 135 (1990), 25-35.

4. S. Shelah, On cardinal invariants of the continuum, Axiomatic Set Theory, Contemp. Math., vol. 31, Amer. Math. Soc., Providence, RI, 1984, pp. 183-207.

Department of Mathematics, University of Michigan, Ann Arbor, Michigan 48109

Current address, Alan Dow: Department of Mathematics, York University, Toronto, Canada M3J 1P3

Current address, Ryszard Frankiewicz: Institute of Mathematics, Polish Academy of Science, Warsaw, Poland 